\title{
Untargeted Metabolomics Analysis Revealed Lipometabolic Disorders in Perirenal Adipose Tissue of Rabbits Subject to a High-Fat Diet
}

\author{
Siqi Xia ${ }^{1,+}$, Jiahao Shao ${ }^{1,+}$, Mauricio A. Elzo ${ }^{2}$, Tao Tang ${ }^{1}$, Yanhong Li ${ }^{1}$, Tianfu Lai ${ }^{1}$, Mingchuan Gan ${ }^{1}$, \\ Yuan Ma ${ }^{1}$, Xianbo Jia ${ }^{3} \mathbb{D}$, Songjia Lai ${ }^{3}$ and Jie Wang ${ }^{3, *}$ \\ 1 College of Animal Science and Technology, Sichuan Agricultural University, Chengdu 611130, China; \\ xiasiqi2020@163.com (S.X.); shaojh1997@163.com (J.S.); m18483220592@163.com (T.T.); \\ lyh81236718@163.com (Y.L.); tf.lai@foxmail.com (T.L.); ganmingchuan1998@163.com (M.G.); \\ manima0916@163.com (Y.M.) \\ 2 Department of Animal Science, University of Florida, Gainesville, FL 32611, USA; maelzo@ufl.edu \\ 3 Farm Animal Genetic Resources Exploration and Innovation Key Laboratory of Sichuan Province, Sichuan \\ Agricultural University, Chengdu 611130, China; jaxb369@sicau.edu.cn (X.J.); laisj5794@163.com (S.L.) \\ * Correspondence: wjie68@sicau.edu.cn \\ + Siqi Xia and Jiahao Shao contributed equally to this work.
}

Citation: Xia, S.; Shao, J.; Elzo, M.A.; Tang, T.; Li, Y.; Lai, T.; Gan, M.; Ma, Y.; Jia, X.; Lai, S.; et al. Untargeted Metabolomics Analysis Revealed Lipometabolic Disorders in Perirenal Adipose Tissue of Rabbits Subject to a High-Fat Diet. Animals 2021, 11, 2289. https://doi.org/10.3390/ani11082289

Academic Editor: Juan José Pascual

Received: 29 June 2021

Accepted: 30 July 2021

Published: 3 August 2021

Publisher's Note: MDPI stays neutral with regard to jurisdictional claims in published maps and institutional affiliations.

Copyright: (c) 2021 by the authors. Licensee MDPI, Basel, Switzerland. This article is an open access article distributed under the terms and conditions of the Creative Commons Attribution (CC BY) license (https:/ / creativecommons.org/licenses/by/ $4.0 /)$.

\begin{abstract}
A high-fat diet (HFD) is widely recognized as a significant modifiable risk for insulin resistance, inflammation, Type 2 diabetes, atherosclerosis and other metabolic diseases. However, the biological mechanism responsible for key metabolic disorders in the PAT of rabbits subject to HFD remains unclear. Here, untargeted metabolomics (LC-MS/MS) combined with liquid chromatography (LC) and high-resolution mass spectrometry (MS) were used to evaluate PAT metabolic changes. Histological observations showed that the adipocytes cells and density of PAT were significantly increased in HFD rabbits. Our study revealed 206 differential metabolites (21 up-regulated and 185 down-regulated); 47 differential metabolites (13 up-regulated and 34 down-regulated), comprising mainly phospholipids, fatty acids, steroid hormones and amino acids, were chosen as potential biomarkers to help explain metabolic disorders caused by HFD. These metabolites were mainly associated with the biosynthesis of unsaturated fatty acids, the arachidonic acid metabolic pathway, the ovarian steroidogenesis pathway, and the platelet activation pathway. Our study revealed that a HFD caused significant lipometabolic disorders. These metabolites may inhibit oxygen respiration by increasing the adipocytes cells and density, cause mitochondrial and endoplasmic reticulum dysfunction, produce inflammation, and finally lead to insulin resistance, thus increasing the risk of Type 2 diabetes, atherosclerosis, and other metabolic syndromes.
\end{abstract}

Keywords: high-fat diet; lipometabolic; metabolomics; perirenal adipose tissue (PAT); rabbit

\section{Introduction}

In recent years, the occurrence rate of obesity has fleetly increased, which poses a risk for many medical diseases, causing concern for many public and health-related professionals. Obesity is a serious medical, social, and economic problem that has caused millions of disabilities, concomitant diseases, and deaths [1,2]. The prevalence of obesity in humans is widespread across all ages and both sexes and can be attributed to the interaction between the environment and physiological factors [3,4]. Nowadays, the universality of a HFD is one of the major contributors to the development of obesity. Moreover, many analyses across various species (rats, mice, and pigs) have indicated that HFD is associated with multiple metabolic syndromes, such as insulin resistance, Type 2 diabetes, cardiovascular disease, fatty liver, hypertension, Alzheimer's disease, and cancer [5-12]. PAT is a kind of white adipose tissue that supports triglyceride (TG) storage for energy demands and endocrine function. Broadly, studies have revealed that PAT plays a significant role in 
controlling lipid mobilization and reproductive function as well as modulating multiple metabolic pathways $[13,14]$. Accumulated evidence shows that the main mechanisms leading to these metabolic diseases include endoplasmic reticulum stress and mitochondrial dysfunction [15], excessive accumulation of metabolites in adipose tissue, imbalance of energy supply and metabolic homeostasis [16], reduction of reverse cholesterol transport [17], aggravation of inflammation and reduction of insulin sensitivity [18]. Above all, one of the most significant and destructive complications is abnormal lipid metabolism, which will definitely worsen in the future [19].

With the popularity of metabolite research, the detection methods of metabolites have also been innovated. Metabonomics is usually used as a tool to discover biomarkers, which can analyze metabolites in biological fluids, tissues, and cells [20]. Moreover, untargeted metabolomics analysis is a kind of metabonomics that can detect and analyze all small molecule metabolites simultaneously without bias. Untargeted metabolomics analysis can evaluate metabolites in detail, explain the categories of metabolites, and examine the relationship between related metabolites in multiple ways [21]. Metabolites maintain homeostasis in response to adverse biological responses. Previous studies have also shown that the use of untargeted metabolomics analysis provides a basis for metabolic syndrome in HFD-fed rats [22,23]. Therefore, untargeted metabolomics is used to identify the phenotype-related metabolites and metabolic pathways to explain the specific functions of metabolites and to understand the physiological effects.

Rabbits are economically important domestic animals raised primarily as a source of animal protein, more recently used as a practical model for obesity-related studies [24]. A previous study reported that the subcutaneous adipose tissue of HFD-fed New Zealand white rabbits after 5 or 10 weeks plays a significant role in obesity-associated systemic lowgrade inflammation [25]. Additionally, there was a study that evaluated changes in blood vessel function, using a HFD-rabbit model [26]. However, the overall metabolic change of PAT in rabbits fed a HFD has yet to be elucidated. Thus, to gain further understanding of the molecular consequences of obesity, we investigated the metabolic change in PAT from the obese rabbit induced by a HFD by using untargeted metabolomics.

\section{Materials and Methods}

\subsection{Ethics Statement}

This study was approved by and conducted in strict accordance with the ethical standards of the Institutional Animal Care and Use Committee of the College of Animal Science and Technology, Sichuan Agricultural University, Sichuan, China.

\subsection{Animals and Experimental Design}

A total of 16 female Tianfu black rabbits (35 days of age) at the teaching farm of the Sichuan Agricultural University were randomly divided into two groups: a control group fed a SND $(n=8)$ and an experimental group fed a HFD (SND plus 10\% lard, $n=8$ ). Detailed information on the feeding procedures can be found in a previous study [27]. Briefly, rabbits from both groups were fed twice a day with free access to water and under a light/dark cycle of $12 \mathrm{~h}$ per day. The room temperature was about 22 to $26^{\circ} \mathrm{C}$. According to the previous research method, the animals were classified as obese [27]. In short, the body weight, body length, bust length and adipose tissue weight were used as the criteria for selecting obese rabbits. After eliminating the substandard rabbits, 6 rabbits were selected from the HFD $(n=6)$ and SND $(n=6)$ groups, respectively, for sampling. After being fed for 5 weeks, the selected rabbits were humanely slaughtered by electrical stunning with exsanguinations for sampling. The rabbit PAT samples were rapidly collected after rabbits were euthanized and stored in Eppendorf tubes at $-80^{\circ} \mathrm{C}$ for subsequent analysis.

\subsection{Histological Examination}

In order to examine the histological changes of PAT, selected rabbits were humanely slaughtered, and PAT was stained with hematoxylin-eosin. In brief, the tissue samples 
were fixed with a $10 \%$ neutral formaldehyde fixator for about $24 \mathrm{~h}$ and then washed with clean water. After that, the tissue samples were dehydrated, embedded in paraffin, and stained with hematoxylin-eosin. Then, the PAT sections (6 to $8 \mu \mathrm{m})$ were collected using a microtome (RM2235, Leica, Nussloch, Germany). Furthermore, an alternative microscope (DM1000, Leica, Nussloch, Germany) was used to capture images at a $200 \times$ field of view. In the end, PAT slices of rabbits from the two groups were analyzed by ImageJ software (National Institutes of Health). We used ImageJ (available at https:/ /imagej.nih.gov/ij/ accessed on 22 July 2021) to quantitatively analyze the staining sections of perirenal adipose tissue in HFD and SND groups, respectively. Briefly, we calculated the PAT cells with more than 4.38 pixels, and calculated the 5 parts (upper, lower, left, right and central regions) of each staining section. Six cells were randomly selected from each region, with a total of 30 cells [28]. Finally, the standard error of mean was calculated, and the unpaired $t$-test was used to compare the differences of PAT between HFD and SND.

\subsection{Sample Preparation}

Approximately $100 \mathrm{mg}$ of the PAT sample from each rabbit was ground into powder in liquid nitrogen. Homogenized samples were resuspended and vortexed by adding a prepared mixture composed of $80 \%$ methanol and $0.1 \%$ formic acid. Then, the samples were placed on ice and incubated for $5 \mathrm{~min}$, followed by centrifugation at $15,000 \times g$ and $4{ }^{\circ} \mathrm{C}$ for $5 \mathrm{~min}$. The methanol concentration of the supernatants was reduced to $53 \%$ by LC-MS/MS grade water. After the samples were transferred to a new Eppendorf tube, they were centrifuged at $15,000 \times \mathrm{g}$ and $4{ }^{\circ} \mathrm{C}$ for $10 \mathrm{~min}$. Lastly, the supernatants were incorporated into the LC-MS/MS system to conduct the LC-MS/MS analysis [29].

\subsection{Ultra-High Performance Liquid Chromatography-Tandem Mass Spectrometry (UHPLC-MS/MS) Analysis}

Chromatographic separation of differential metabolites was accomplished by injecting PAT samples into a Hypesil Gold HPLC column $(2.1 \mathrm{~mm} \times 100 \mathrm{~mm}, 1.9 \mu \mathrm{m})$ (Thermo Fisher, Waltham, MA, USA) using a Vanquish UHPLC System (Thermo Fisher, Bremen, Germany) coupled with a Q Exactive HF-X Hybrid Quadrupole-Orbitrap Mass Spectrometer (Thermo Fisher, Germany) located at Novogene Co., Ltd. (Beijing, China). In detail, the Hypesil Gold HPLC column was kept at $40{ }^{\circ} \mathrm{C}$ with a flow rate of $0.2 \mathrm{~mL} / \mathrm{min}$ using a 17 min linear gradient. The eluent $\mathrm{B}$ (Methanol) was the same for both positive and negative polarity modes. However, the eluent A differed for the positive ( $0.1 \%$ FA in Water) and negative (5 mM ammonium acetate, $\mathrm{pH} 9.0$ ) polarity modes. The solvent gradient was set as follows: $2 \% \mathrm{~B}, 1.5 \mathrm{~min} ; 2$ to $100 \% \mathrm{~B}, 12.0 \mathrm{~min} ; 100 \% \mathrm{~B}, 14.0 \mathrm{~min} ; 100$ to $2 \% \mathrm{~B}, 14.1 \mathrm{~min} ; 2 \% \mathrm{~B}, 17 \mathrm{~min}$. The Q Exactive HF-X Hybrid Quadrupole-Orbitrap Mass Spectrometer was operated in both positive and negative electron spray ionization $(\mathrm{ESI}+) /(\mathrm{ESI}-)$ modes with a spray voltage of $3.2 \mathrm{kV}$. Moreover, the settings were $320{ }^{\circ} \mathrm{C}$ for the capillary temperature, 40 arb for the sheath gas flow rate, and 10 arb for the aux flow rate.

\subsection{Data Processing and Analysis}

The raw data files generated by UHPLC-MS/MS were processed with Compound Discoverer 3.1 (CD 3.1, Thermo Fisher) to determine the peak value for each metabolite. The detailed parameters were set as follows: (a) the retention time tolerance was $0.2 \mathrm{~min}$; (b) the actual mass tolerance was 5 ppm; (c) the signal intensity tolerance was 30\%; (d) the signal ratio was 3 ; and (e) the minimum intensity was 100,000 . Then, the peak values were matched with the mzCloud (https://www.mzcloud.org/ accessed on 24 August 2020), mzVault, and MassList databases to obtain the exact and relative quantitative results. To better observe the inter-group distributions and otherness, the program metaX was used for PCA and PLS-DA. The quality of the PLS-DA models was assessed, using R2X, R2Y, and Q2. R2X and R2Y are fractions of sum of squares explained by a given principal component. Q2 represents the predictive ability of the PLS-DA model. Permutation tests with 200 permutations were used to validate the models. 


\subsection{Identification and Analysis of Metabolites}

Differently expressed metabolites were identified based on the value of VIP, the $p$ value, and fold change (FC). In detail, the metabolites with variable importance in projection (VIP) $>1, p$-value $<0.05$, and FC $\geq 2$ or FC $\leq 0.5$ were considered to be differential metabolites. Moreover, volcano plots were used to filter metabolites of interest, using log2 (FC) and $-\log 10$ ( $p$-value). Differential metabolites were annotated with the Human Metabolome Database (HMDB, https:/ / hmdb.ca/metabolites, accessed on 24 August 2020), and LIPID MAPS (http:/ / www.lipidmaps.org/ accessed on 24 August 2020) databases, respectively, to obtain a systematized overview. The differential metabolites-related metabolic pathways and physiological functions were explored by using the Kyoto Encyclopedia of Genes and Genomes (KEGG) database (https:/ / www.genome.jp/kegg/pathway.html, accessed on 24 August 2020). A differential metabolic pathway was considered to be significantly enriched when the observed frequency of a metabolite $(x / n)>$ expected frequency of a metabolite $(\mathrm{y} / n)$, and $p$ value $<0.05$. Clustering heat maps were plotted with $\mathrm{R}$ package Pheatmap (https:/ / cran.r-project.org/web / packages/pheatmap/pheatmap.pdf, accessed on 24 August 2020).

\subsection{Statistical Analysis}

In this study, statistical software available in R (R version R-3.4.3) (MathSoft, Auckland, New Zealand), Python (Python 2.7.6 version) (The Python Software Foundation, Amsterdam, The Netherlands) and CentOS Linux (CentOS release 6.6) (Ctrl IQ) were used to analyze the metabolic data. A univariate $t$-test was applied to calculate the statistical significance, and only $p$-values $<0.05$ were considered statistically significant. The $p$ values were adjusted for multiple hypotheses by using the Benjamini-Hochberg procedure, controlling for the false discovery rate (FDR) (https: / / cran.r-project.org, accessed on 30 July 2021).

\section{Results}

\subsection{PAT Histological Observations}

The hematoxylin-eosin-stained PAT samples in the SND and HFD groups showed a normal cell structure; however, a difference in the cell size and number was observed (Figure 1). Compared with the SND group, the number of PAT cells in the HFD was significantly increased (100.3 \pm 1.453 vs. $77.33 \pm 2.906, p=0.0021)$; however, the area of PAT cells was significantly decreased $(779.45 \pm 30.822 \mu \mathrm{m}$ vs. $1759.59 \pm 100.502 \mu \mathrm{m}$, $p<0.0001)$.

HFD

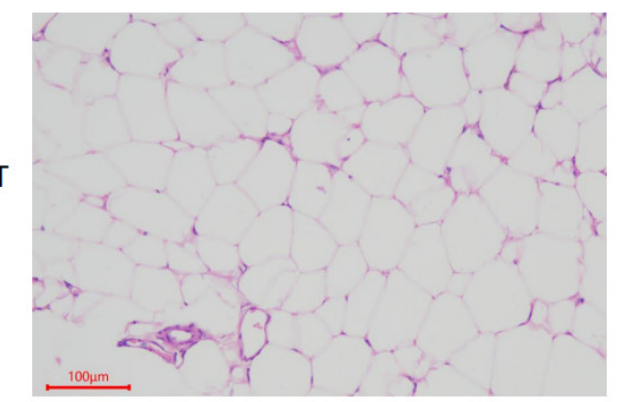

SND

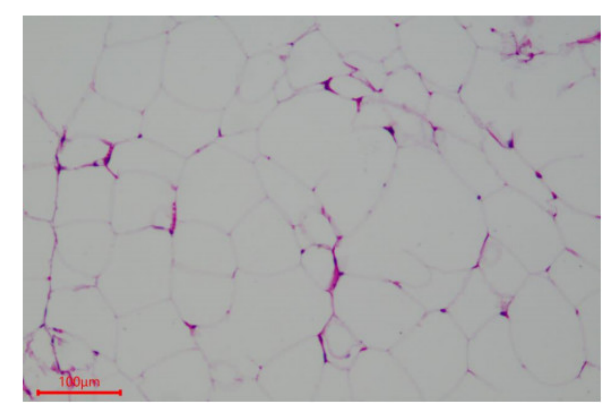

Figure 1. Hematoxylin-eosin staining of PAT in female rabbits fed SND $(n=6)$ and $\operatorname{HFD}(n=6)$.

\subsection{Quality Control of Metabolomics Data}

The Pearson correlation coefficients between the positive and negative quality control (QC) samples are shown in Figure S1. The Pearson correlation coefficient between the QC samples is higher than 0.991 for both ESI+ and ESI-, indicating that the stability of detection and quality of data are excellent. The PCA score plots show that the distributions 
of PAT metabolites from the SND and HFD groups differ (Figure 2A,B). All the PLS-DA score plots for the SND and HFD groups (Figure 2C,D) are within the $95 \%$ confidence interval, except for the 17th and 19th samples. Samples \#17 and \#19 are not within the confidence interval. For the following analysis, these two samples are not included in the experimental analysis. The values of the PLS-DA statistics are $\mathrm{R} 2 \mathrm{Y}=0.92$ and $\mathrm{Q} 2=0.67$ for the ESI+ data, and R2Y $=0.91, \mathrm{Q} 2=0.66$ for the ESI - data. These R2Y and Q2 values indicate that the PAT metabolic differences between SND and HFD rabbits were quite significant. Permutation tests were used to prevent over-fitting of the PLS-DA models. The validation included 200 random permutation tests, which generated intercepts of R2 $=0.76$ and $\mathrm{Q} 2=-0.69$ for the ESI + data and $\mathrm{R} 2=0.68$ and $\mathrm{Q} 2=-0.91$ for the ESI - data (Figure 2E,F), indicating that the PLS-DA models are credible and without over-fitting. Thus, the PLS-DA models show an excellent predictive ability and reliability to determine significant PAT metabolic disturbances in HFD rabbits.

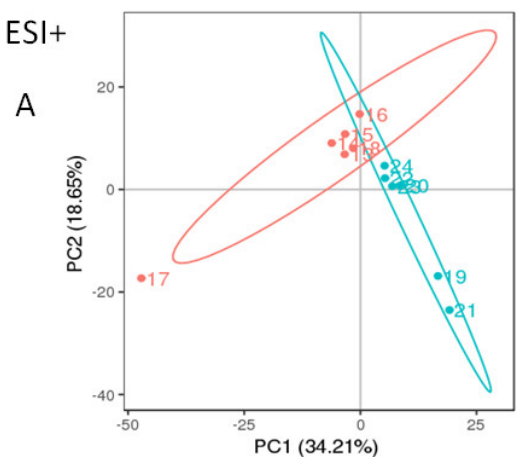

$\mathrm{ESI+}$

C

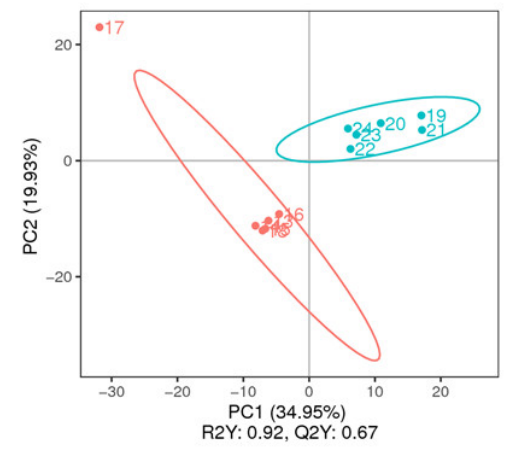

$\mathrm{ESI+}$

HFD vs. SND

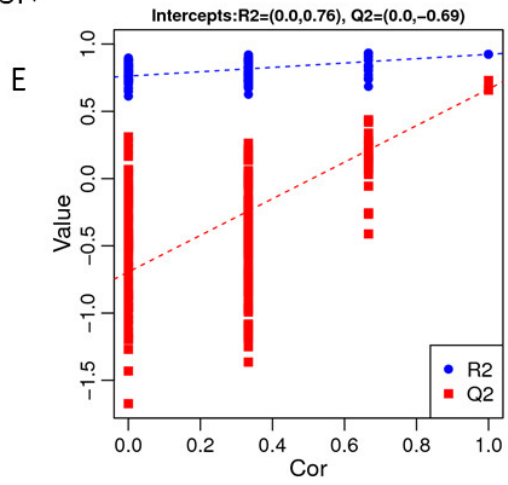

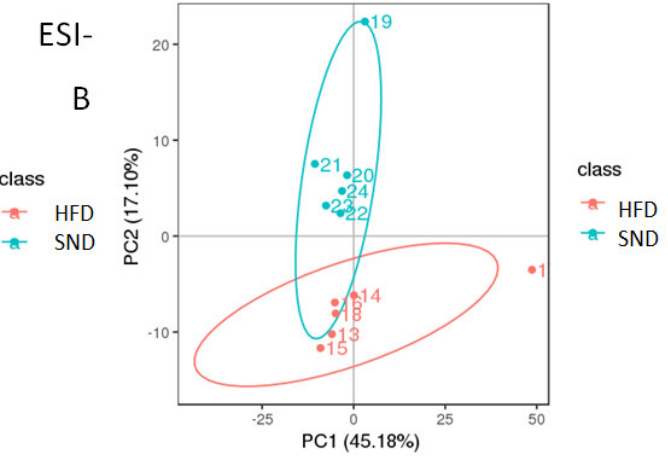

ESI-

D

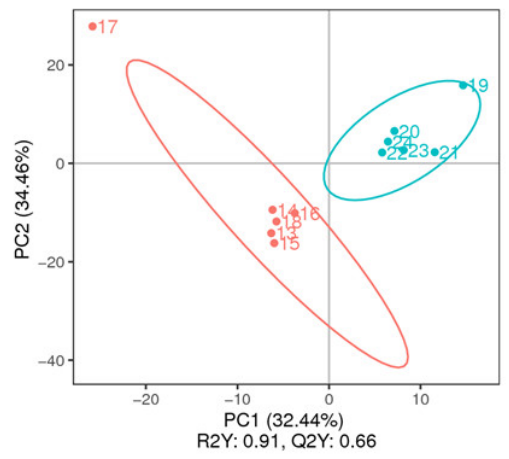

ESI-

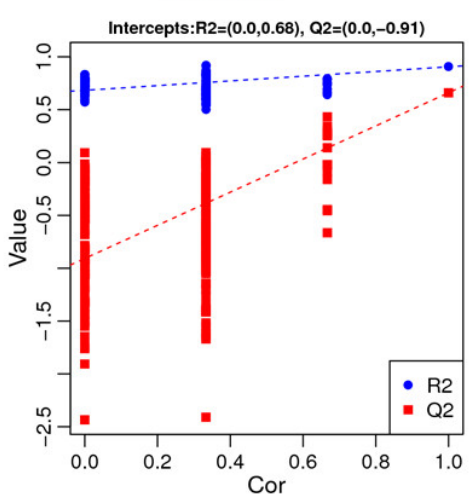

Figure 2. Quality control of metabolomics data. PCA score plots for SND $(n=6)$ and HFD $(n=6)$ groups in the positive (A) and negative (B) ion modes. PLS-DA score plots for SND $(n=6)$ and HFD $(n=6)$ groups in the positive $(\mathbf{C})$ and negative $(\mathbf{D})$ ion modes. Permutation tests from PLS-DA models for SND $(n=6)$ and HFD $(n=6)$ groups in the positive $(\mathbf{E})$ and negative $(\mathbf{F})$ ion modes. 


\subsection{Differential Metabolites Analysis}

The VIP of the first principal component of the PLS-DA model combined with the $p$ value was used to ascertain metabolites with differential expression in the SND and HFD groups. The thresholds were VIP $>1.0, \mathrm{FC}>1.5$ or FC $<0.665$, and $p$ value $<0.05$ [30-32]. The value of FDR is shown in Tables S1 and S2. A total of 206 metabolites were detected, of which 21 were up-regulated, and 185 were down-regulated (Tables S1 and S2). The volcano maps in Figure 3A,B visually show the overall distribution of the differential metabolites. Based on KEGG, HMDB and LIPID MAPS databases, a total of 50 metabolic pathways were detected in the HFD group, compared with the SND group in the two modes, of which fatty acids, phospholipids, and sterol lipids were identified as the main metabolites (Tables S3 and S4). Then, on this basis, the metabolites chosen as potential biomarkers to help explain metabolic disorders caused by HFD were those with VIP values above 1 and a $p$ value below 0.05 [33]. A total of 47 metabolites were detected, of which 13 were significantly up-regulated, and 34 were significantly down-regulated (Table 1). In addition, hierarchical clustering analysis was conducted on the obtained metabolites of the two groups to obtain the differences of metabolic expression patterns between and within the same comparison group. So, the cluster heat map also showed the distribution of differential metabolites between the HFD and SND (Figure 3C,D). These results further illustrate that the metabolism of PAT was disturbed by HFD.

Table 1. Metabolites with significant difference were analyzed by LC-MS/MS to identify potential biomarkers of interest.

\begin{tabular}{|c|c|c|c|c|c|}
\hline Metabolites' Name & Formula & $\mathbf{M}^{1}$ & RT $^{2}$ & VIP ${ }^{3}$ & Trend ${ }^{4}$ and $p$ Value (HFD vs. SND) \\
\hline PC $(2: 0 / 16: 1)$ & $\mathrm{C} 26 \mathrm{H} 50 \mathrm{NO} 8 \mathrm{P}$ & 595.3491 & 13.322 & 1.563966765 & $\downarrow^{* *}$ \\
\hline PC (2:0/16:0) & $\mathrm{C} 26 \mathrm{H} 52 \mathrm{NO} 8 \mathrm{P}$ & 597.36482 & 13.637 & 1.678708 & $\downarrow *$ \\
\hline $\mathrm{PC}(2: 0 / 16: 2)$ & $\mathrm{C} 26 \mathrm{H} 48 \mathrm{NO} 8 \mathrm{P}$ & 533.312 & 13.083 & 1.257585 & $\downarrow * *$ \\
\hline PC $(7: 0 / 8: 0)$ & C23H46NO8P & 495.296 & 13.64 & 1.709003 & $\downarrow * *$ \\
\hline $\mathrm{PC}(8: 0 / 8: 0)$ & $\mathrm{C} 24 \mathrm{H} 48 \mathrm{NO} 8 \mathrm{P}$ & 509.3122 & 12.994 & 1.482654 & $\downarrow *$ \\
\hline PC $(14: 0 \mathrm{e} / 3: 0)$ & C25H52NO7P & 509.3481 & 15.092 & 1.797233 & $\downarrow * *$ \\
\hline PC $(14: 0 \mathrm{e} / 5: 0)$ & C27H56NO7P & 537.3799 & 15.604 & 1.563913 & $\downarrow * *$ \\
\hline PC (14:0e/15:0) & C37H76NO7P & 677.5239 & 15.723 & 1.590351 & $\downarrow * *$ \\
\hline PC $(15: 1 / 18: 2)$ & C41H76NO8P & 741.5327 & 16.04 & 1.638059 & $\downarrow *$ \\
\hline PC (16:0e/13:0) & C37H76NO7P & 660.4966 & 15.72 & 1.602117 & $\downarrow * *$ \\
\hline PC (16:0e/20:4) & C44H82NO7P & 767.5806 & 16.279 & 1.665696 & $\downarrow *$ \\
\hline PC $(16: 2 / 18: 5)$ & C42H70NO8P & 709.5431 & 15.675 & 1.384283 & $\downarrow * *$ \\
\hline PC $(17: 2 / 22: 6)$ & C47H78NO8P & 815.5423 & 15.998 & 1.250684 & $\uparrow *$ \\
\hline PC $(18: 1 / 18: 2)$ & C44H82NO8P & 783.5737 & 16.245 & 1.218645 & $\downarrow *$ \\
\hline PC $(18: 1 / 19: 2)$ & C45H84NO8P & 797.5907 & 16.194 & 1.176725 & $\downarrow^{*}$ \\
\hline PC $(18: 2 / 19: 2)$ & C45H82NO8P & 795.5781 & 15.76 & 2.130609 & $\downarrow^{2} *$ \\
\hline PC (18:4e/20:5) & C46H76NO7P & 785.5238 & 15.649 & 1.666749 & $\uparrow * *$ \\
\hline PC (18:4e/22:6) & C48H78NO7P & 811.5374 & 15.781 & 1.790827 & $\downarrow * *$ \\
\hline PC $(19: 2 / 18: 4)$ & C45H78NO8P & 791.5449 & 16.305 & 1.457723 & ** \\
\hline PC $(20: 2 / 20: 3)$ & C48H86NO8P & 835.5957 & 15.534 & 1.313735 & $\downarrow *$ \\
\hline PC (22:3e/18:4) & C48H84NO7P & 817.594 & 15.407 & 1.361675 & $\downarrow * *$ \\
\hline PC $(20: 3 / 20: 3)$ & C48H84NO8P & 833.5871 & 15.567 & 1.34977 & $\downarrow *$ \\
\hline LPC 15:0 & C23H48NO7P & 481.3167 & 14.571 & 1.951213 & $\downarrow *$ \\
\hline LPC 19:0 & C27H56NO7P & 597.40091 & 15.577 & 2.003144 & $\downarrow^{*}$ \\
\hline PE (2:0/16:2) & $\mathrm{C} 23 \mathrm{H} 42 \mathrm{NO} 8 \mathrm{P}$ & 491.2647 & 13.081 & 1.33104 & $\downarrow^{2 * *}$ \\
\hline PE $(5: 0 / 13: 1)$ & C23H44NO8P & 493.2803 & 13.564 & 1.864231 & $\downarrow * *$ \\
\hline PE $(2: 0 / 18: 1)$ & C25H48NO8P & 521.31072 & 14.223 & 1.958401 & $\downarrow * *$ \\
\hline PE (17:1/18:1) & C40H76NO8P & 729.53193 & 16.438 & 2.004323 & $\downarrow * *$ \\
\hline PE (18:2/18:2) & C41H74NO8P & 739.5142 & 16.165 & 1.086091 & $\downarrow *$ \\
\hline PE (18:2/20:4) & C43H74NO8P & 763.50059 & 15.487 & 1.856928 & $\downarrow^{* *}$ \\
\hline PE (20:3/20:4) & C45H76NO8P & 789.5234 & 15.926 & 1.857655 & $\uparrow *$ \\
\hline LPE 16:1 & C21H42NO7P & 451.2696 & 14.594 & 1.583397 & $\downarrow * *$ \\
\hline LPE 17:0 & $\mathrm{C} 22 \mathrm{H} 46 \mathrm{NO} 7 \mathrm{P}$ & 467.3011 & 14.966 & 1.396921 & $\downarrow *$ \\
\hline LPE $17: 1$ & $\mathrm{C} 22 \mathrm{H} 44 \mathrm{NO} 7 \mathrm{P}$ & 465.2855 & 14.621 & 2.160675 & $\downarrow *$ \\
\hline LPE 20:4 & C25H44NO7P & 501.2857 & 14.407 & 1.723687 & ${ }^{v} *$ \\
\hline LPS $18: 2$ & C24H44 N O9 P & 521.27548 & 13.894 & 1.839273 & $\uparrow * *$ \\
\hline LPS 18:0 & C24 H48NO9 P & 525.30639 & 14.412 & 1.545101 & $\downarrow *$ \\
\hline PG $(18: 2 / 20: 4)$ & C44H75O10P & 794.51067 & 15.605 & 1.039475 & $\uparrow *$ \\
\hline $\mathrm{SM}(\mathrm{d} 19: 1 / 19: 0)$ & $\mathrm{C} 43 \mathrm{H} 87 \mathrm{~N} 2 \mathrm{O} 6 \mathrm{P}$ & 758.6277 & 15.612 & 1.201017 & $\uparrow *$ \\
\hline Arachidonic acid & $\mathrm{C} 2 \mathrm{OH} 32 \mathrm{O} 2$ & 304.23985 & 14.263 & 1.235326 & $\uparrow *$ \\
\hline Adrenic acid & $\mathrm{C} 22 \mathrm{H} 36 \mathrm{O} 2$ & 332.27124 & 14.649 & 1.732588 & $\uparrow *$ \\
\hline Docosapentaenoic acid & $\mathrm{C} 22 \mathrm{H} 34 \mathrm{O} 2$ & 330.2556 & 15.208 & 2.095414 & $\uparrow * *$ \\
\hline Docosahexaenoic acid & $\mathrm{C} 22 \mathrm{H} 32 \mathrm{O} 2$ & 328.23993 & 14.209 & 1.90978 & $\uparrow * *$ \\
\hline Methyltestosterone & $\mathrm{C} 2 \mathrm{OH} 30 \mathrm{O} 2$ & 302.22456 & 14.414 & 2.348698 & $\uparrow * *$ \\
\hline 2-Hydroxyestradiol & $\mathrm{C} 18 \mathrm{H} 24 \mathrm{O} 3$ & 288.17234 & 10.513 & 1.100492 & $\downarrow * *$ \\
\hline
\end{tabular}


Table 1. Cont.

\begin{tabular}{|c|c|c|c|c|c|}
\hline Metabolites' Name & Formula & $\mathbf{M}^{1}$ & $\mathbf{R T}^{2}$ & VIP $^{3}$ & Trend ${ }^{4}$ and $p$ Value (HFD vs. SND) \\
\hline Epitestosterone & $\mathrm{C} 19 \mathrm{H} 28 \mathrm{O} 2$ & 288.2084 & 13.648 & 1.387 & $\downarrow^{*}$ \\
\hline Cholecalciferol & $\mathrm{C} 27 \mathrm{H} 44 \mathrm{O}$ & 384.3388 & 15.714 & 1.61838 & $\downarrow^{* *}$ \\
\hline 4-Pyridoxic acid & $\mathrm{C} 8 \mathrm{H} 9 \mathrm{NO} 4$ & 183.0533 & 7.482 & 1.766454 & $\downarrow^{* *}$ \\
\hline L-Methionine & C5H11NO2S & 149.0511 & 1.964 & 1.568533 & $\uparrow *$ \\
\hline
\end{tabular}

NOTE: ${ }^{1} \mathrm{~m}$ : molecular weight. ${ }^{2}$ RT: retention time. ${ }^{3}$ VIP: the importance projection of variables is used to reflect the contribution of quantitative value of each sample to the difference, generally, VIP $>1 .{ }^{4}$ Trend and $p$ value (HFD vs. SND): ${ }^{*} p<0.05 ;{ }^{* *} p<0.01$.

HFD vs. SND

A

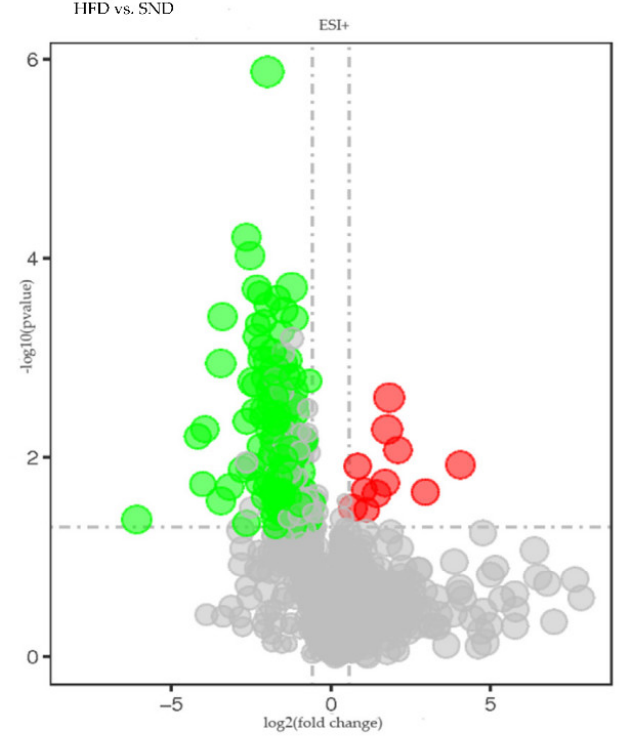

ESI+

C

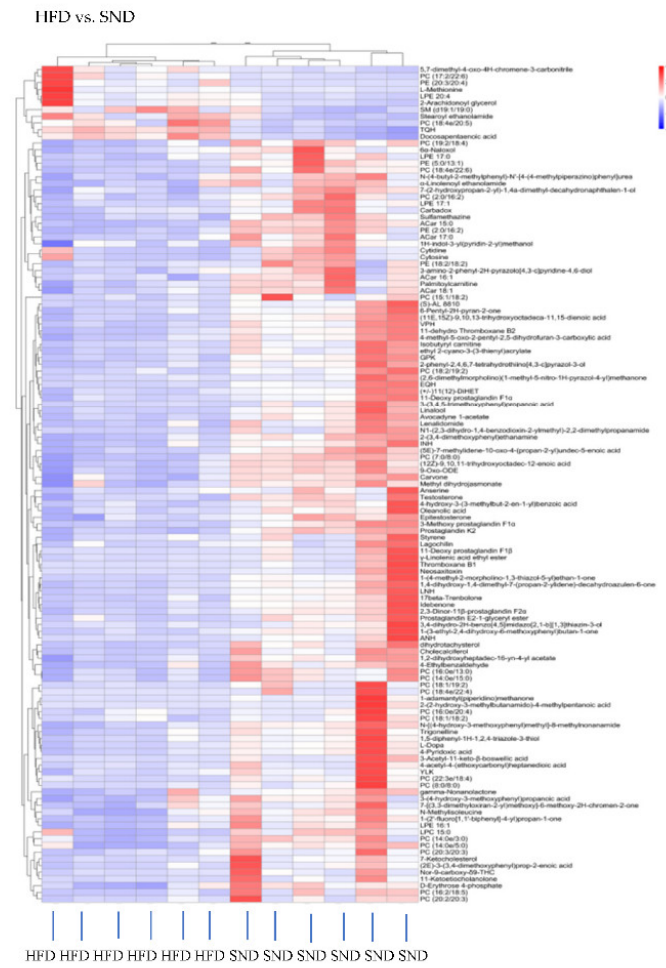

HFD vs. SND
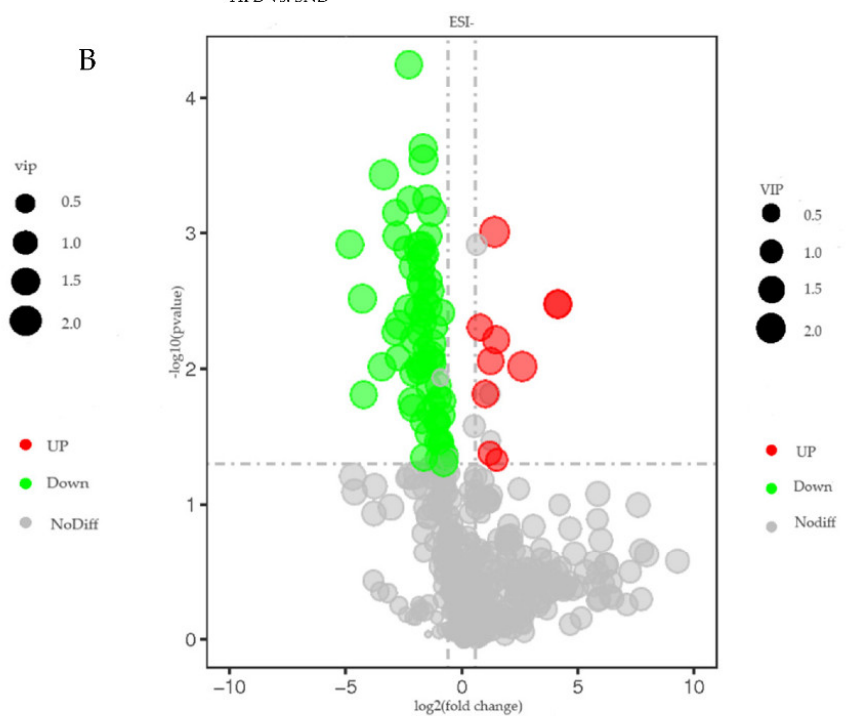

ESI- HFD vs. SND

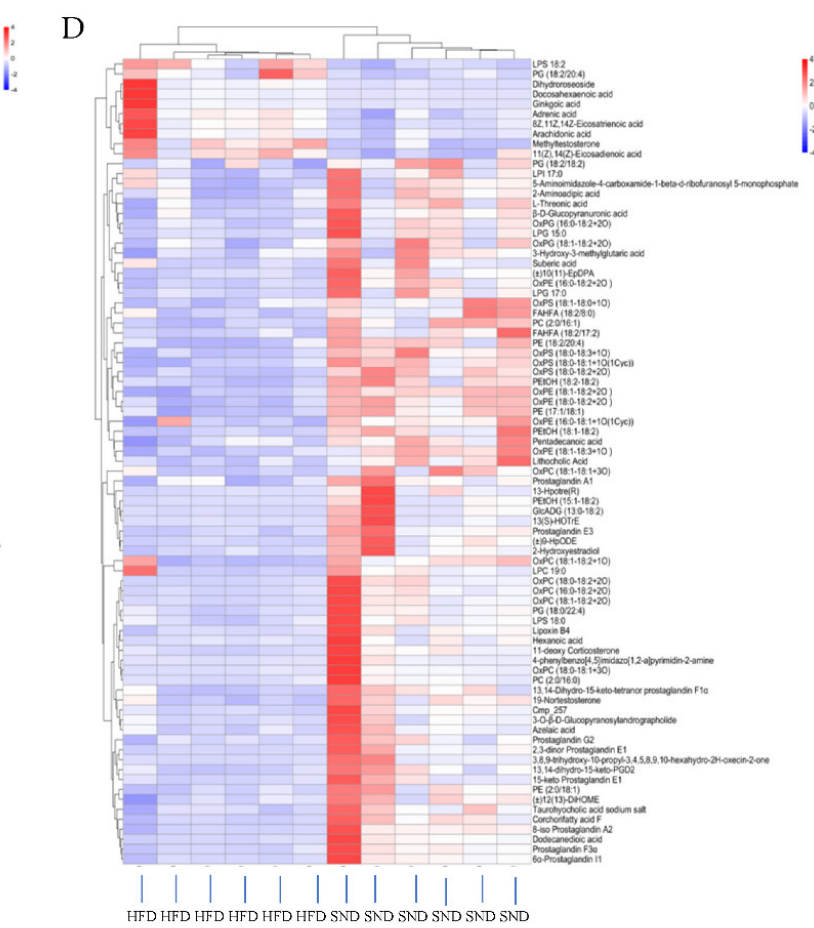

Figure 3. Differential metabolites analysis. Volcano maps showing the overall distribution of differential metabolites in rabbit groups fed SND $(n=6)$ and $\operatorname{HFD}(n=6)$ in the positive $(\mathbf{A})$ and negative $(\mathbf{B})$ ion modes. Red circles represent significantly higher numbers of metabolites, and green circles indicate significantly lower numbers of metabolites in HFD $(n=6)$ than in SND $(n=6)$ rabbit groups. Heat map representing a hierarchical clustering of positive (C) and negative (D) ion mode of differential metabolites between HFD and SND. Shades of red and blue represent an increase or reduction of a metabolite, respectively, relative to median metabolite levels (see color scale). A = HFD, $a=$ SND. 


\subsection{Metabolic Pathway Analysis}

To study the changed pathways induced by HFD, an exhaustive KEGG pathway analysis was conducted. Significance was ascertained, using a hypergeometric test with a threshold $p$ value $\leq 0.05$ to filter out pathways with $p$ values higher than 0.05 . In detail, the major identified metabolic pathways were platelet activation, arachidonic acid metabolism, ovarian steroidogenesis, biosynthesis of unsaturated fatty acids, ferroptosis, and vitamin B6 metabolism (Tables S3 and S4). The top 20 signaling pathways are shown in the positive (Figure 4A) and negative (Figure 4B) KEGG enrichment plots obtained with MetaboAnalyst. Among these pathways, the vitamin B6 metabolic signaling pathway, arachidonic acid metabolic pathway, biosynthesis of unsaturated fatty acids, platelet activation, serotonergic synapse, ovarian steroidogenesis, and ferroptosis were highly enriched in the HFD rabbit group. Given that PAT is an organ for lipid storage and metabolism, we picked some pathways associated with the lipid cycle for a more detailed analysis of the metabolites (Figure 4C). These metabolites are mainly phospholipids, fatty acids, steroid hormones, and L-methionine, implying that these molecules may be the key molecules in the development of obesity.

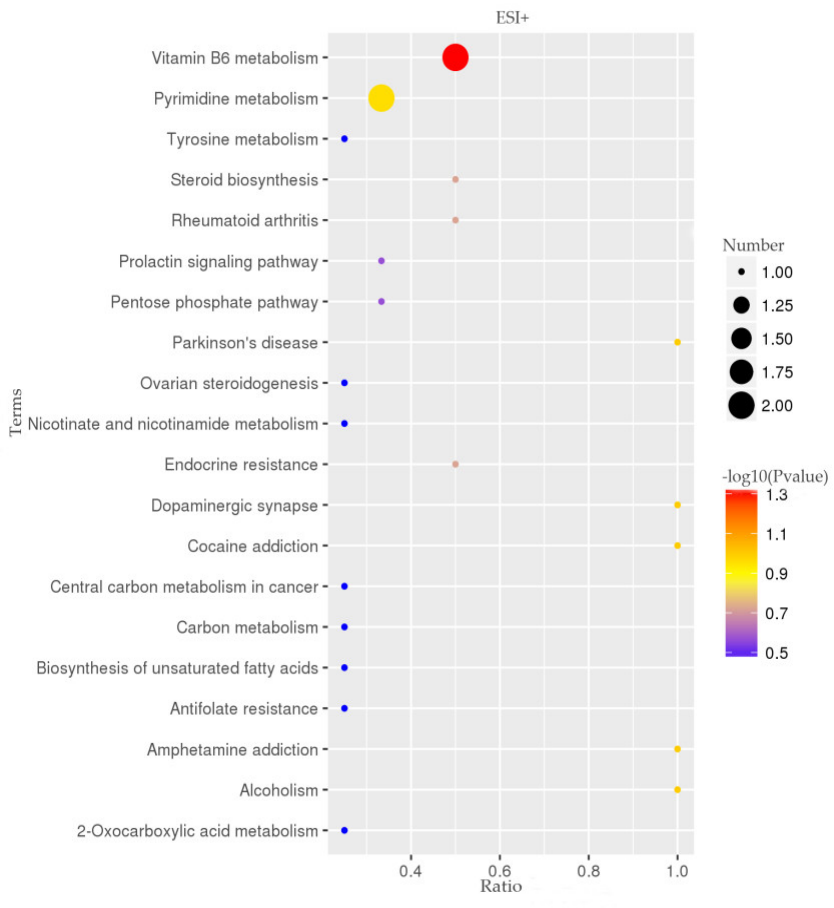

(A)

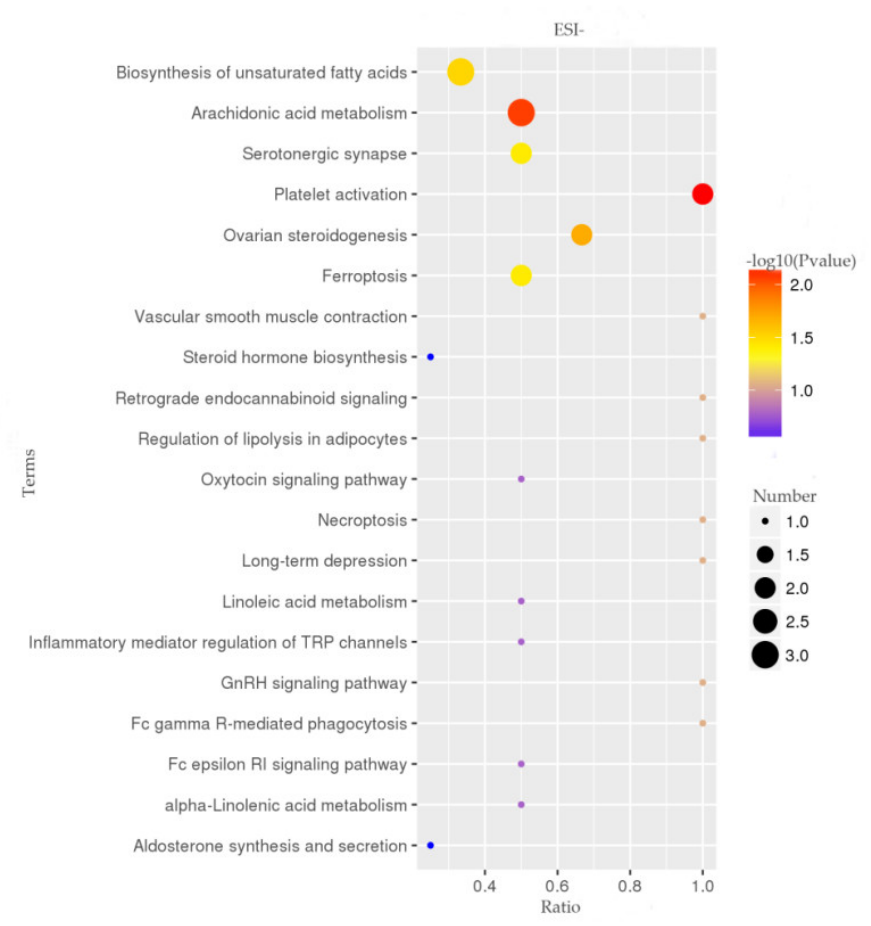

(B)

Figure 4. Cont. 


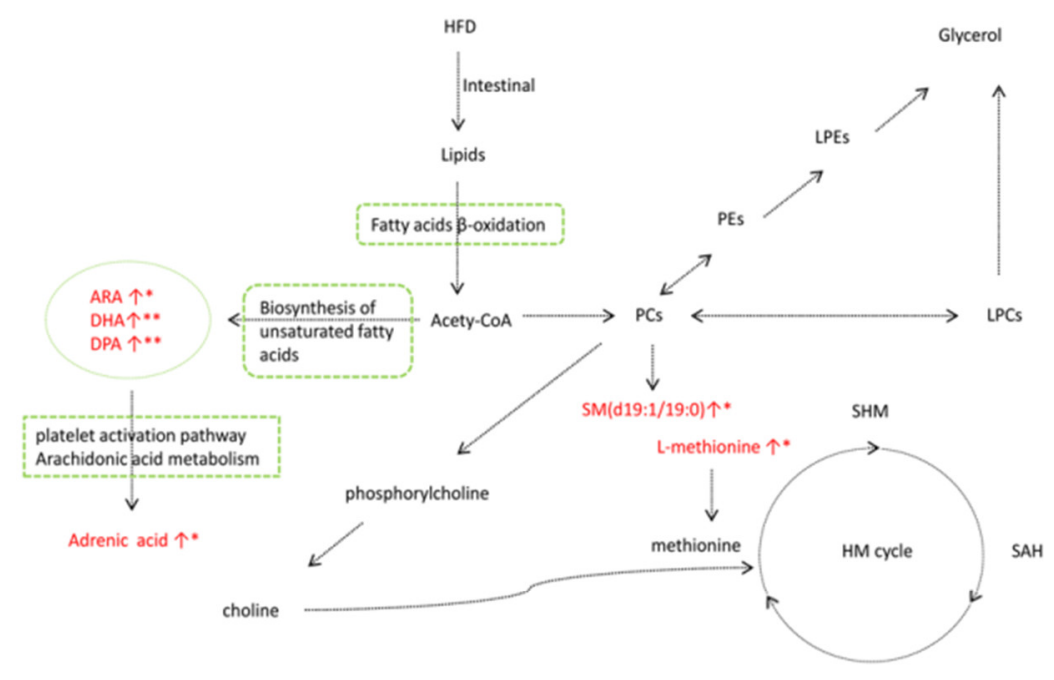

$\mathrm{HCY}$

\begin{tabular}{|cl|}
\multicolumn{1}{c}{$\mathrm{PCs}$} \\
\hline $\mathrm{PC}(2: 0 / 16: 1)$ & $\downarrow * *$ \\
$\mathrm{PC}(2: 0 / 16: 2)$ & $\downarrow * *$ \\
$\mathrm{PC}(7: 0 / 8: 0)$ & $\downarrow * *$ \\
$\mathrm{PC}(14: 0 \mathrm{e} / 3: 0)$ & $\downarrow^{* *}$ \\
$\mathrm{PC}(14: 0 \mathrm{e} / 5: 0)$ & $\downarrow^{* *}$ \\
$\mathrm{PC}(14: 0 \mathrm{e} / 15: 0)$ & $\downarrow^{* *}$ \\
$\mathrm{PC}(16: 0 \mathrm{e} / 13: 0)$ & $\downarrow^{* *}$ \\
$\mathrm{PC}(16: 2 / 18: 5)$ & $\downarrow^{* *}$ \\
$\mathrm{PC}(17: 2 / 22: 6)$ & $\uparrow^{*}$ \\
$\mathrm{PC}(18: 2 / 19: 2)$ & $\downarrow * *$ \\
$\mathrm{PC}(18: 4 \mathrm{e} / 20: 5)$ & $\uparrow * *$ \\
$\mathrm{PC}(18: 4 \mathrm{e} / 22: 6)$ & $\downarrow * *$ \\
$\mathrm{PC}(19: 2 / 18: 4)$ & $\downarrow * *$ \\
$\mathrm{PC}(22: 3 \mathrm{e} / 18: 4)$ & $\downarrow * *$ \\
\hline
\end{tabular}
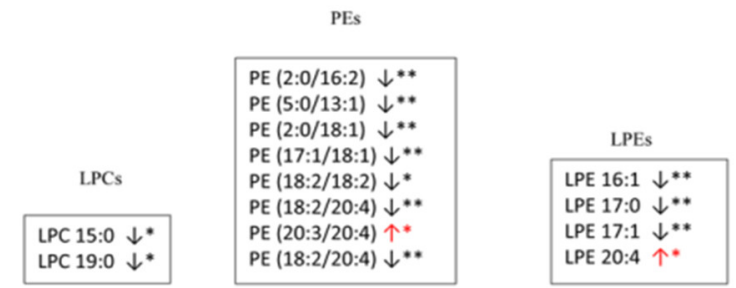

(C)

Figure 4. Metabolic pathway analysis. KEGG positive (A) and negative (B) pathway enrichment plots obtained with MetaboAnalyst. Metabolic pathways are represented by circles, circle size and color shade are based on pathway impact and $p$ value (red being the most significant). (C) The network of metabolic pathways associated with the lipid cycle. Metabolite levels are shown in color: red represents increased levels, and black represents decreased or undetected levels. Trend and $p$ value (HFD vs. SND): ${ }^{*} p<0.05 ;{ }^{* *} p<0.01$.

\section{Discussion}

Feeding a HFD is the most common method for animal models of obesity, thus HFD continues to be an indispensable method for discovering mechanisms of metabolic syndromes [34]. Similarly, untargeted metabolomics, an effective method to measure metabolites, plays an important role in understanding the physiological functions of metabolites and the potential causes of metabolic disorders [20].

Feeding rabbits with a HFD will result in damage to the normal function of PAT, destruction of the balance between lipid formation and degradation, and overaccumulation of lipids in PAT. The LC-MS/MS metabolite analyses showed that the PAT lipid cycle in rabbits fed a HFD was disturbed, resulting in significant changes in the levels of phospholipids, fatty acids, steroid hormones, and L-methionine (Figure 4C). Concordant with previous studies, heat maps also showed significant differences in metabolites between HFD-fed rats, compared with control (normal diet), and this long-term HFD diet led to the development of obesity-related insulin resistance syndrome in rats [35]. Similarly, feeding mice with a HFD caused a metabolic imbalance that resulted in metabolic disorders, such as insulin resistance and nonalcoholic steatohepatitis [36]. Hematoxylin-eosin staining of PAT showed differences between HFD and SND groups. To further confirm that there is such a difference between them, we used ImageJ to quantify the cells of PAT. The results certainly showed a significant increase in the number of HFD cells and a significant decrease in the 
area, indicating an increase in cell density in the HFD group, which is consistent with a previous study on human visceral fat [37]. It may cause the absorption of oxygen in lipids to be inhibited, resulting in an imbalance of lipid metabolites and lipid accumulation.

The symptoms of obesity closely resemble the spectrum of metabolic changes in PAT, including phospholipids, fatty acids, steroid hormones, and amino acids, among which phospholipids and lysophosphatides are the most abundant metabolites. Phospholipids are the main components of plasma membranes, including phosphatidylethanolamines (PEs) and phosphatidylcholines (PCs), which are precursors of lysophosphatidylethanolamine (LPEs) and lysophosphatidylcholines (LysoPCs/LPCs), respectively [38]. In our study, PCs and PEs were the most frequently found phospholipids (most of them downregulated in HFD rabbits), whereas levels of all LPCs were reduced. Concerning the 14 PCs and 8 PEs in PAT, some PCs, such as PC (18:4e/20:5) and PC (17:2/22:6), increased in HFD rabbits, which was similar to a previous study in humans, where some PCs were shown to be significantly higher in the obese group than that in the control group [39]. The level of PE (18:2/18:2) was lower in mice fed a HFD than for mice fed a normal diet [40], consistent with our results. Further, the level of LPCs (LPC 15:0, LPC 19:0) decreased in rabbits fed a HFD for 4 weeks, which was partially in agreement with a decrease in plasma in human obesity and Type 2 diabetes [41], and a low-abundance of LPCs in the serum of hyperlipidemic mice fed a HFD [42]. PE is methylated to PC [43]. Changes in phospholipid levels can inhibit calcium ion transport and affect the transfer of phospholipids between the endoplasmic reticulum (ER) and mitochondria, inducing ER stress and mitochondrial dysfunction, which will decrease fatty acid oxidation and acetyl CoA levels [15]. Of course, this is only the speculation made by previous studies that phospholipids may act as a bad metabolite for the characterization of mitochondrial dysfunction. In our next experiments, we will focus on the setting of mitochondria to further improve our research. In addition, an imbalance in the PC/PE ratio will affect the mitochondria-associated ER membranes, leading to an excessive accumulation of sphingomyelin (SM) in the ER, inducing the activation of PKC, inhibiting the activity of AKT and disrupting the energy supply and metabolic homeostasis [16]. SM is produced by group transfer in phosphatidylcholine combined with the associated skeleton, which is closely related to sphingomyelin synthase (SMS). A significant increase in SM may reduce reverse cholesterol transport, increasing the risk of atherosclerosis lesions and other metabolic diseases [17]. Further, as an important signal molecule, reduced levels of LPC combined with some cell-specific G-coupled protein receptors can cause an increase in insulin secretion through glucose stimulation, which will damage $\beta$ cell function, leading to insulin resistance, stimulate the production of adipocytes, and aggravate the risk of obesity and other diseases $[15,44]$. These are the factors that may cause insulin resistance. The decrease in LPCs in rabbits from this study may be related to an increase in insulin resistance; thus, LPCs could be considered as potential biomarkers for metabolic diseases caused by obesity due to HFD. Our results here indicate that SM levels were significantly up-regulated in the HFD, compared to the SND rabbit groups, in agreement with reports of obesity and insulin sensitivity in obese adult humans [45] and a study on the plasma metabolic fingerprints of atherosclerosis rabbits [46]. These results suggest that changes in phospholipids levels may reduce insulin sensitivity, lead to insulin resistance, and increase the risk of atherosclerosis.

Arachidonic acid (ARA) and adrenic acid are omega- 6 polyunsaturated fatty acids. In the current study, levels of ARA were higher in the HFD than in the SND rabbit group, in agreement with the significant increase in the serum ARA levels in rats fed a HFD [47]. According to our identification results, ARA was the main metabolite of the arachidonic acid metabolic, ovarian steroidogenesis, biosynthesis of unsaturated fatty acids, and ferroptosis metabolism pathways. However, a 'one-to-many' type of relationship was pointed out between metabolic pathways that were annotated and identified compounds. ARA matched 18 associated metabolic pathways, which showed that at least ARA was comparatively important for PAT. The most important metabolic pathway for ARA in this study was platelet activation $(p<0.01)$. Under normal circumstances, the release of fatty acids in 
PAT is strictly controlled to meet energy requirements. Conversely, metabolic disorders cause an excessive release of fatty acids relative to the tissue requirements. It is widely accepted that disturbances in the fatty acid metabolism will lead to increased inflammatory signaling, which is a central factor in insulin resistance [18]. ARA promotes the production of several prostaglandins, which are associated with lipopolysaccharide (LPS)-induced inflammation [48]. It is hypothesized that the increase in ARA is associated with PAT metabolic disorders and may induce inflammation to further produce insulin resistance. Previous studies have shown that the concentration of body fat and adipocytokines in the (HFD + ARA) group was significantly increased after six weeks of induction [49]. Further, excessive levels of ARA can cause oxidative stress and activate pro-inflammatory signals that induce endoplasmic reticulum (ER) stress, leading to insulin resistance [50,51]. Hence, ARA can be used as an indicator of PAT metabolic disorders in obese patients consuming HFD. Endogenous adrenal acid is produced by ARA, and it is mainly oxidized in the peroxisome. Consistent with the results here, the plasma levels of adrenal acid were found to be up-regulated in an adipose hepatitis model, and primarily caused by instability of peroxidase $\beta$-oxidation [52]. Docosahexaenoic acid (DHA) and docosapentaenoic acid (DPA) are long-chain omega-3 polyunsaturated fatty acids (PUFAs). The levels of DPA and DHA were significantly elevated in PAT from HFD-fed rabbits, compared to SND-fed rabbits. The higher levels of DPA and DHA in the HFD rabbit group disagreed with the significantly lower levels of DPA and DHA in 12-week-old rats fed a HFD, relative to rats in the control group. Further, a strong positive association existed between the reduced levels of these two metabolites and the insulin sensitivity index [35]. A possible explanation is that a higher concentration of n-3 PUFAs inhibits the release of free fatty acids from PAT [53], which in turn inhibits the inflammatory signaling pathway and decreases the risk of insulin resistance, thus playing a protective role. In addition, DHA and DPA have strong anti-inflammatory effects and can activate peroxisome, thus increasing insulin sensitivity [53,54]. The levels of DPA and DHA in the HFD rabbit group were significantly increased, which may indicate a protective effect. Therefore, rabbits in the HFD group may have insulin resistance and other metabolic syndromes, but they may also produce metabolites, such as DHA and DPA, to protect them against adverse factors. However, no free fatty acids were detected in the plasma/serum of the HFD rabbits; thus, additional research is needed.

Steroid hormones, such as testosterone, 2-hydroxyestradiol, and epitestosterone, were also found in this study. These hormones play vital roles in the production and metabolic function of adipose tissue via hormone receptors. Levels of testosterone, 2hydroxyestradiol, and epitestosterone were significantly lower in PAT from the HFD than the SND rabbit group. The hormone 2-hydroxyestradiol has strong inhibitory effects on NADPH during lipid peroxidation in rat microsomes. In addition, lipid peroxides depend on specific ions at the initial stage and are strongly inhibited by oxygen absorption [55], which may inhibit the PAT lipid metabolic pathway and cause lipid accumulation. Changes in testosterone were the result of rats being fed HFD to induce insulin resistance [56], strongly suggesting that changes in the rabbit testosterone levels are due to rabbits being fed HFD, inducing insulin resistance. Furthermore, a study showed that testosterone gradually increased in visceral fat rather than in subcutaneous adipose tissue in human females [37]. This result agreed with the significantly increased adipocytes cells and density of PAT in HFD rabbits, relative to SND rabbits. Thus, changes in the 2-hydroxyestradiol and testosterone levels in this study may have led to lipid accumulation by increasing adipocytes cells and the density of PAT and inhibiting the absorption of oxygen by lipid metabolism. However, the specific mechanism of steroid hormones in PAT needs further study.

L-methionine produces methionine, and it functions not only as an essential amino acid, but also as a physiological effector [57]. We found higher levels of L-methionine in HFD than in SND rabbits. The methionine cycle provides methyl units for various reactions, including methylation in lipids. The S-adenosine methionine (SAM) is used as a major 
methyl donor molecule, and it is synthesized from the essential amino acid methionine [58]. Choline, produced by phosphatidylcholine, and the subsequent substances produced by choline oxidation, such as betaine, can not only help to adjust cell volume, but also act as methyl donors in the homocysteine-methionine (HM) cycle, transporting excess fatty acids to corresponding organelles for metabolism [59]. Methionine supplementation increases the homocysteine (Hcy) concentration and is associated with vitamin B6. Therefore, in our study, one possible explanation is the excessive accumulation of fatty acids in PAT of rabbits fed with a HFD, and the increase in the L-methionine level, thus further increasing the level of Hcy, and finally disturbing the HM cycle. However, previous studies have reported that high circulating Hcy concentrations are related to an elevated risk of atherosclerosis, steatohepatitis and lipid metabolic disturbances [60,61]. Changes in the HM cycle after feeding lean Iberian sows with a HFD were associated with obesity-related diseases and Type 2 diabetes [62], indicating that the higher levels of L-methionine may be related to atherosclerotic diseases and Type 2 diabetes by affecting the HM cycle.

\section{Conclusions}

Histological examination and untargeted metabonomics analysis revealed that rabbits fed a HFD exhibited PAT metabolic disorders, affecting unsaturated fatty acid synthesis, and the arachidonic acid metabolic, ovarian steroidogenesis, and platelet activation pathways. Phospholipids and excessive levels of ARA may cause mitochondrial dysfunction and inflammation, and induce ER stress, leading to insulin resistance. Steroid hormones may inhibit oxygen absorption by increasing the adipocytes cells and density of PAT. Lmethionine may increase the risk of Type 2 diabetes and atherosclerosis by affecting the HM cycle. Contrary to previous studies, we found significantly elevated levels of DHA and DPA, which are inversely associated with obesity in both humans and animals. This aspect merits further research. The metabolic changes and biomarkers identified in this study may serve as a foundation for future therapeutic interventions against lipometabolic disorders.

Supplementary Materials: The following are available online at https:/ /www.mdpi.com/article/10 $.3390 /$ ani11082289/s1. Figure S1: Pearson correlation coefficient between positive and negative QC samples. The left is positive ion mode and the right is negative ion mode. Table S1: Total positive metabolites with difference in PAT of rabbits fed either a SND $(n=6)$ or HFD $(n=6)$ was analyzed by LC-MS/MS. Table S2: Total negative metabolites with significant difference in PAT of rabbits fed either SND $(n=6)$ or HFD $(n=6)$ was analyzed by LC-MS/MS. Table S3: KEGG enrichment analysis of differential positive metabolites in PAT of rabbits fed either a SND $(n=6)$ or HFD $(n=6)$. Table S4: KEGG enrichment analysis of differential negative metabolites in PAT of rabbits fed either a SND $(n=6)$ or $\operatorname{HFD}(n=6)$.

Author Contributions: Conceived and designed the study: J.W., X.J., S.L. Collected data and conducted the research: T.T., Y.L., T.L., M.G., Y.M. Wrote the paper: S.X., J.S.; M.A.E. revised the paper. All authors have read and agreed to the published version of the manuscript.

Funding: Our study was funded by the key research and development project of Sichuan Province: high quality and characteristic rabbit breeding materials and methods innovation and new variety breeding (2021YFYZ0033).

Institutional Review Board Statement: This study was approved by and conducted in strict accordance with the ethical standards of the Institutional Animal Care and Use Committee of the College of Animal Science and Technology, Sichuan Agricultural University, Sichuan, China.

Informed Consent Statement: Not applicable.

Data Availability Statement: All data generated or analyzed during this study are included.

Conflicts of Interest: The authors declare no conflict of interest. 


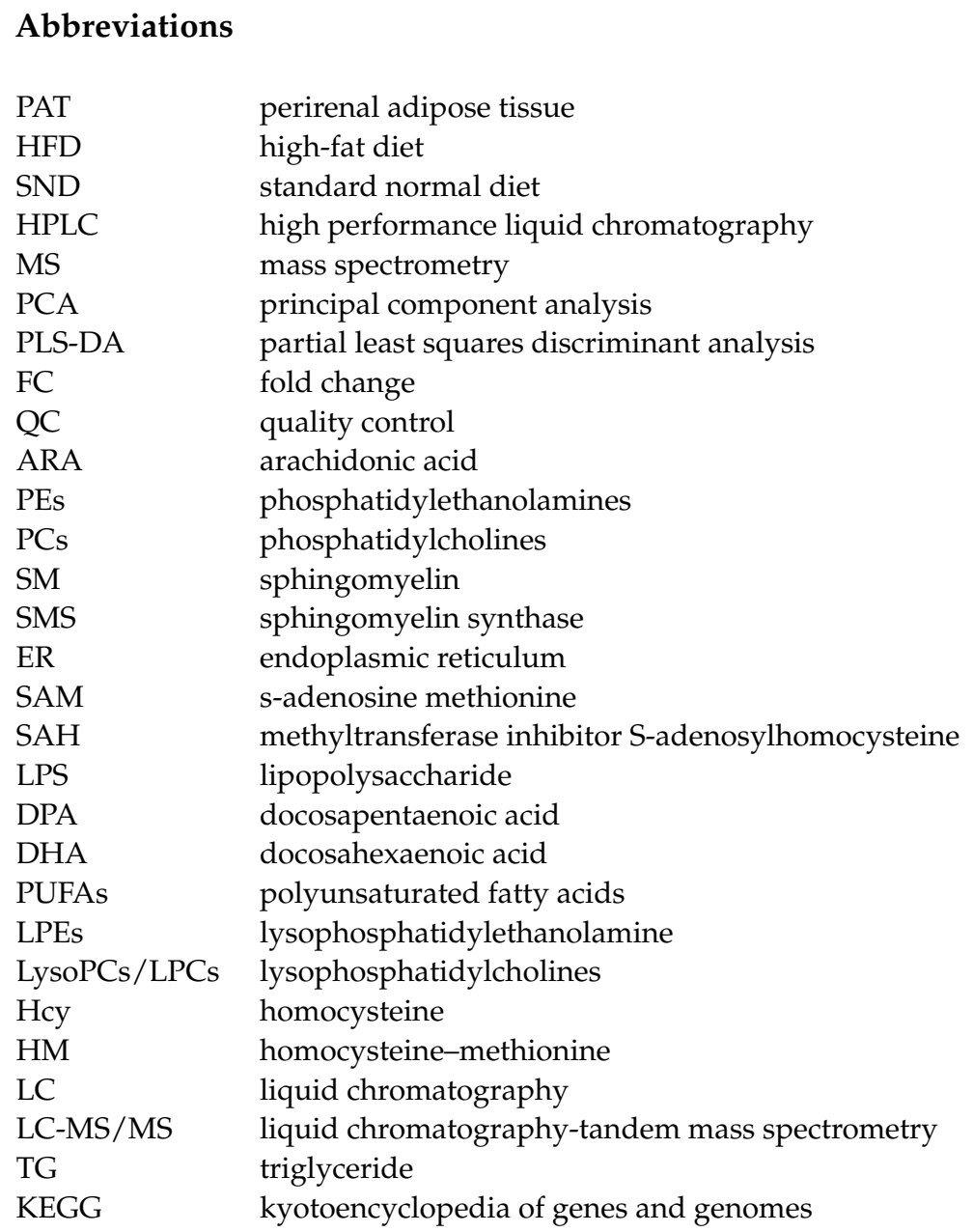

\section{References}

1. Ng, M.; Fleming, T.; Robinson, M.; Thomson, B.; Graetz, N.; Margono, C.; Mullany, E.C.; Biryukov, S.; Abbafati, C.; Abera, S.F.; et al. Global, regional, and national prevalence of overweight and obesity in children and adults during 1980-2013: A systematic analysis for the Global Burden of Disease Study 2013. Lancet 2014, 384, 766-781. [CrossRef]

2. Wyatt, S.B.; Winters, K.P.; Dubbert, P.M. Overweight and Obesity: Prevalence, Consequences, and Causes of a Growing Public Health Problem. Am. J. Med Sci. 2006, 331, 166-174. [CrossRef]

3. Reilly, J.J.; El-Hamdouchi, A.; Diouf, A.; Monyeki, M.A.; A Somda, S. Determining the worldwide prevalence of obesity. Lancet 2018, 391, 1773-1774. [CrossRef]

4. Elobeid, M.A.; Allison, D.B. Putative environmental-endocrine disruptors and obesity: A review. Curr. Opin. Endocrinol. Diabetes Obes. 2008, 15, 403-408. [CrossRef] [PubMed]

5. McLaughlin, T.; Ackerman, S.E.; Shen, L.; Engleman, E. Role of innate and adaptive immunity in obesity-associated metabolic disease. J. Clin. Investig. 2017, 127, 5-13. [CrossRef] [PubMed]

6. Bhupathiraju, S.N.; Hu, F.B. Epidemiology of Obesity and Diabetes and Their Cardiovascular Complications. Circ. Res. 2016, 118, 1723-1735. [CrossRef]

7. O'Sullivan, J.; Lysaght, J.; Donohoe, C.L.; Reynolds, J.V. Obesity and gastrointestinal cancer: The interrelationship of adipose and tumour microenvironments. Nat. Rev. Gastroenterol. Hepatol. 2018, 15, 699-714. [CrossRef] [PubMed]

8. Liu, Z.; Patil, I.Y.; Jiang, T.; Sancheti, H.; Walsh, J.P.; Stiles, B.L.; Yin, F.; Cadenas, E. High-Fat Diet Induces Hepatic Insulin Resistance and Impairment of Synaptic Plasticity. PLoS ONE 2015, 10, e0128274. [CrossRef]

9. Picone, P.; Di Carlo, M.; Nuzzo, D. Obesity and Alzheimer's disease: Molecular bases. Eur. J. Neurosci. 2020, 52, 3944-3950. [CrossRef]

10. Lira, F.S.; Rosa, J.C.; A Cunha, C.; Ribeiro, E.B.; Nascimento, C.O.D.; Oyama, L.M.; Mota, J.F. Supplementing alpha-tocopherol (vitamin $\mathrm{E}$ ) and vitamin $\mathrm{D}_{3}$ in high fat diet decrease IL-6 production in murine epididymal adipose tissue and 3T3-L1 adipocytes following LPS stimulation. Lipids Health Dis. 2011, 10, 37. [CrossRef] [PubMed]

11. Wang, Q.; Yuan, J.; Yu, Z.; Lin, L.; Jiang, Y.; Cao, Z.; Zhuang, P.; Whalen, M.J.; Song, B.; Wang, X.-J.; et al. FGF21 Attenuates High-Fat Diet-Induced Cognitive Impairment via Metabolic Regulation and Anti-inflammation of Obese Mice. Mol. Neurobiol. 2018, 55, 4702-4717. [CrossRef] 
12. Rodríguez, R.R.; González-Bulnes, A.; Garcia-Contreras, C.; Rodríguez-Rodríguez, A.E.; Astiz, S.; Vazquez-Gomez, M.; Pesantez, J.L.; Isabel, B.; Salido-Ruiz, E.; González, J.; et al. The Iberian pig fed with high-fat diet: A model of renal disease in obesity and metabolic syndrome. Int. J. Obes. 2020, 44, 457-465. [CrossRef]

13. Luo, L.; Liu, M. Adipose tissue in control of metabolism. J. Endocrinol. 2016, 231, R77-R99. [CrossRef]

14. Bocquier, F.; Bonnet, M.; Faulconnier, Y.; Guerre-Millo, M.; Martin, P.; Chilliard, Y. Effects of photoperiod and feeding level on perirenal adipose tissue metabolic activity and leptin synthesis in the ovariectomized ewe. Reprod. Nutr. Dev. 1998, 38, 489-498. [CrossRef]

15. Cheng, H.; Gang, X.; He, G.; Liu, Y.; Wang, Y.; Zhao, X.; Wang, G. The Molecular Mechanisms Underlying MitochondriaAssociated Endoplasmic Reticulum Membrane-Induced Insulin Resistance. Front. Endocrinol. 2020, 11, 592129. [CrossRef]

16. Blouin, C.M.; Prado, C.; Takane, K.K.; Lasnier, F.; Garcia-Ocana, A.; Ferre, P.; Dugail, I.; Hajduch, E. Plasma Membrane Subdomain Compartmentalization Contributes to Distinct Mechanisms of Ceramide Action on Insulin Signaling. Diabetes 2010, 59, 600-610. [CrossRef]

17. Meikle, J.; Summers, S.A. Sphingolipids and phospholipids in insulin resistance and related metabolic disorders. Nat. Rev. Endocrinol. 2017, 13, 79-91. [CrossRef]

18. Solinas, G.; Becattini, B. JNK at the crossroad of obesity, insulin resistance, and cell stress response. Mol. Metab. 2017, 6, 174-184. [CrossRef] [PubMed]

19. Fu, S.; Yang, L.; Li, P.; Hofmann, O.; Dicker, L.; Hide, W.; Lin, X.; Watkins, S.M.; Ivanov, A.R.; Hotamisligil, G.S. Aberrant lipid metabolism disrupts calcium homeostasis causing liver endoplasmic reticulum stress in obesity. Nature 2011, 473, 528-531. [CrossRef] [PubMed]

20. Johnson, C.; Ivanisevic, J.; Siuzdak, G. Metabolomics: Beyond biomarkers and towards mechanisms. Nat. Rev. Mol. Cell Biol. 2016, 17, 451-459. [CrossRef] [PubMed]

21. Zamboni, N.; Saghatelian, A.; Patti, G.J. Defining the metabolome: Size, flux, and regulation. Mol. Cell 2015, 58, 699-706. [CrossRef] [PubMed]

22. Zhu, H.; Wang, Z.; Wu, Y.; Jiang, H.; Zhou, F.; Xie, X.; Wang, R.; Hua, C. Untargeted metabonomics reveals intervention effects of chicory polysaccharide in a rat model of non-alcoholic fatty liver disease. Int. J. Biol. Macromol. 2019, 128, 363-375. [CrossRef] [PubMed]

23. Gowda, S.G.B.; Gao, Z.J.; Chen, Z.; Abe, T.; Hori, S.; Fukiya, S.; Ishizuka, S.; Yokota, A.; Chiba, H.; Hui, S.-P. Untargeted Lipidomic Analysis of Plasma from High.-fat Diet.-induced Obese Rats Using UHPLC-Linear Trap Quadrupole-Orbitrap MS. Anal. Sci. 2020, 36, 821-828. [CrossRef] [PubMed]

24. Martínez-Álvaro, M.; Hernández, P.; Agha, S.; Blasco, A. Correlated responses to selection for intramuscular fat in several muscles in rabbits. Meat Sci. 2018, 139, 187-191. [CrossRef] [PubMed]

25. Lowry, J.E.; Tumurbaatar, B.; D’Agostino, C.; Main, E.; Wright, T.; Dillon, E.L.; Saito, T.B.; Porter, C.; Andersen, C.R.; Brining, D.L.; et al. Effect of high-fat diet on peripheral blood mononuclear cells and adipose tissue in early stages of diet-induced weight gain. Br. J. Nutr. 2019, 122, 1359-1367. [CrossRef] [PubMed]

26. Alarcon, G.; Roco, J.; Medina, M.; Medina, A.; Peral, M.; Jerez, S. High fat diet-induced metabolically obese and normal weight rabbit model shows early vascular dysfunction: Mechanisms involved. Int. J. Obes. 2018, 42, 1535-1543. [CrossRef] [PubMed]

27. Shao, J.; Wang, J.; Li, Y.; Elzo, M.A.; Tang, T.; Lai, T.; Ma, Y.; Gan, M.; Wang, L.; Jia, X.; et al. Growth, behavioural, serum biochemical and morphological changes in female rabbits fed high-fat diet. J. Anim. Physiol. Anim. Nutr. 2021, 105, 345-353. [CrossRef]

28. Testa, V.; De Santis, N.; Scotto, R.; Della Giustina, P.; Desideri, L.F.; Cellerino, M.; Cordano, C.; Inglese, M.; Uccelli, A.; Vagge, A.; et al. Corneal epithelial dendritic cells in patients with multiple sclerosis: An in vivo confocal microscopy study. J. Clin. Neurosci. 2020, 81, 139-143. [CrossRef]

29. Want, E.J.; O’Maille, .G.; Smith, C.; Brandon, T.R.; Uritboonthai, W.; Qin, C.; Trauger, A.S.A.; Siuzdak, G. Solvent-Dependent Metabolite Distribution, Clustering, and Protein Extraction for Serum Profiling with Mass Spectrometry. Anal. Chem. 2006, 78, 743-752. [CrossRef]

30. Heischmann, S.; Quinn, K.; Cruickshank-Quinn, C.; Liang, L.-P.; Reisdorph, R.; Reisdorph, N.; Patel, M. Exploratory Metabolomics Profiling in the Kainic Acid Rat Model Reveals Depletion of 25-Hydroxyvitamin D3 during Epileptogenesis. Sci. Rep. 2016, 6, 31424. [CrossRef]

31. Haspel, J.A.; Chettimada, S.; Shaik, R.S.; Chu, J.-H.; Raby, B.A.; Cernadas, M.; Carey, V.; Process, V.; Hunninghake, G.M.; Ifedigbo, E.; et al. Circadian rhythm reprogramming during lung inflammation. Nat. Commun. 2014, 5, 4753. [CrossRef]

32. Sreekumar, A.; Poisson, L.M.; Rajendiran, T.M.; Khan, A.P.; Cao, Q.; Yu, J.; Laxman, B.; Mehra, R.; Lonigro, R.J.; Li, Y.; et al. Metabolomic profiles delineate potential role for sarcosine in prostate cancer progression. Nature 2009, 457, 910-914. [CrossRef]

33. Ma, N.; Liu, X.; Kong, X.; Li, S.; Jiao, Z.; Qin, Z.; Dong, P.; Yang, Y.; Li, J. Feces and liver tissue metabonomics studies on the regulatory effect of aspirin eugenol eater in hyperlipidemic rats. Lipids Health Dis. 2017, 16, 240. [CrossRef]

34. Kleinert, M.; Clemmensen, C.; Hofmann, S.; Moore, M.C.; Renner, S.; Woods, S.C.; Huypens, P.; Beckers, J.; de Angelis, M.H.; Schürmann, A.; et al. Animal models of obesity and diabetes mellitus. Nat. Rev. Endocrinol. 2018, 14, 140-162. [CrossRef]

35. Huang, J.-P.; Cheng, M.-L.; Hung, C.-Y.; Wang, C.-H.; Hsieh, P.-S.; Shiao, M.-S.; Chen, J.-K.; Li, D.-E.; Hung, L.-M. Docosapentaenoic acid and docosahexaenoic acid are positively associated with insulin sensitivity in rats fed high-fat and high-fructose diets. J. Diabetes 2017, 9, 936-946. [CrossRef] [PubMed] 
36. Cai, X.; Fang, C.; Hayashi, S.; Hao, S.; Zhao, M.; Tsutsui, H.; Nishiguchi, S.; Sheng, J. Pu-erh tea extract ameliorates high-fat dietinduced nonalcoholic steatohepatitis and insulin resistance by modulating hepatic IL-6/STAT3 signaling in mice. J. Gastroenterol. 2016, 51, 819-829. [CrossRef] [PubMed]

37. Wiik, A.; Andersson, D.P.; Brismar, T.; Chanpen, S.; Dhejne, C.; Ekström, T.J.; Flanagan, J.N.; Holmberg, M.; Kere, J.; Lilja, M.; et al. Metabolic and functional changes in transgender individuals following cross-sex hormone treatment: Design and methods of the GEnder Dysphoria Treatment in Sweden (GETS) study. Contemp. Clin. Trials Commun. 2018, 10, 148-153. [CrossRef]

38. Facchini, L.; Losito, I.; Cianci, C.; Cataldi, T.R.T.R.I.; Palmisano, F. Structural characterization and profiling of lyso-phospholipids in fresh and in thermally stressed mussels by hydrophilic interaction liquid chromatography-electrospray ionization-Fourier transform mass spectrometry. Electrophoresis 2016, 37, 1823-1838. [CrossRef]

39. Whiley, L.; Sen, A.; Heaton, J.; Proitsi, P.; García-Gómez, D.; Leung, R.; Smith, N.; Thambisetty, M.; Kloszewska, I.; Mecocci, P. Evidence of altered phosphatidylcholine metabolism in Alzheimer's disease. Neurobiol. Aging 2014, 35, 271-278. [CrossRef] [PubMed]

40. Kim, H.-J.; Kim, J.H.; Noh, S.; Hur, H.J.; Sung, M.J.; Hwang, J.-T.; Park, J.H.; Yang, H.J.; Kim, M.-S.; Kwon, D.Y.; et al. Metabolomic Analysis of Livers and Serum from High-Fat Diet Induced Obese Mice. J. Proteome Res. 2011, 10, 722-731. [CrossRef]

41. Barber, M.N.; Risis, S.; Yang, C.; Meikle, P.; Staples, M.; Febbraio, M.A.; Bruce, C.R. Plasma Lysophosphatidylcholine Levels Are Reduced in Obesity and Type 2 Diabetes. PLoS ONE 2012, 7, e41456. [CrossRef] [PubMed]

42. Jin, S.; Song, C.; Li, S.; Zhang, Y.; Chen, C.; Zhou, X.; Xu, Y.; Feng, Y.; Zhang, Z.; Jiang, H. Preventive effects of turmeric on the high-fat diet-induced hyperlipidaemia in mice associated with a targeted metabolomic approach for the analysis of serum lysophosphatidylcholine using LC-MS/MS. J. Funct. Foods 2014, 11, 130-141. [CrossRef]

43. Ye, C.; Sutter, B.M.; Wang, Y.; Kuang, Z.; Tu, B.P. A Metabolic Function for Phospholipid and Histone Methylation. Mol. Cell 2017, 66, 180-193.e8. [CrossRef]

44. Pories, W.J.; Dohm, G.L. Diabetes: Have We Got It All Wrong?: Hyperinsulinism as the culprit: Surgery provides the evidence. Diabetes Care 2012, 35, 2438-2442. [CrossRef]

45. Hanamatsu, H.; Ohnishi, S.; Sakai, S.; Yuyama, K.; Mitsutake, S.; Takeda, H.; Hashino, S.; Igarashi, Y. Altered levels of serum sphingomyelin and ceramide containing distinct acyl chains in young obese adults. Nutr. Diabetes 2014, 4, e141. [CrossRef]

46. Liu, Y.-T.; Peng, J.-B.; Jia, H.-M.; Cai, D.-Y.; Zhang, H.-W.; Yu, C.-Y.; Zou, Z.-M. UPLC-Q/TOF MS standardized Chinese formula Xin-Ke-Shu for the treatment of atherosclerosis in a rabbit model. Phytomedicine 2014, 21, 1364-1372. [CrossRef]

47. Liu, T.-W.; Heden, T.D.; Morris, E.M.; Fritsche, K.L.; Vieira-Potter, V.J.; Thyfault, J.P. High-Fat Diet Alters Serum Fatty Acid Profiles in Obesity Prone Rats: Implications for In Vitro Studies. Lipids 2015, 50, 997-1008. [CrossRef]

48. Sonnweber, T.; Pizzini, A.; Nairz, M.; Weiss, G.; Tancevski, I. Arachidonic Acid Metabolites in Cardiovascular and Metabolic Diseases. Int. J. Mol. Sci. 2018, 19, 3285. [CrossRef]

49. Mak, I.L.; Lavery, P.; Agellon, S.; Rauch, F.; Murshed, M.; A Weiler, H. Arachidonic acid exacerbates diet-induced obesity and reduces bone mineral content without impacting bone strength in growing male rats. J. Nutr. Biochem. 2019, 73, 108226. [CrossRef]

50. Hotamisligil, G.S. Endoplasmic Reticulum Stress and the Inflammatory Basis of Metabolic Disease. Cell 2010, 140, 900-917. [CrossRef] [PubMed]

51. Johnson, A.M.; Olefsky, J.M. The Origins and Drivers of Insulin Resistance. Cell 2013, 152, 673-684. [CrossRef] [PubMed]

52. Horas, H.; Nababan, S.; Kawano, Y.; Kobayashi, T.; Yoshida, M.; Azuma, T. Adrenic acid as an inflammation enhancer in non-alcoholic fatty liver disease. Arch. Biochem. Biophys. 2017, 623, 64-75. [CrossRef] [PubMed]

53. Guo, X.-F.; Li, X.; Shi, M.; Li, D. n-3 Polyunsaturated Fatty Acids and Metabolic Syndrome Risk: A Meta-Analysis. Nutrients 2017, 9, 703. [CrossRef] [PubMed]

54. Serhan, C.N. Pro-resolving lipid mediators are leads for resolution physiology. Nature 2014, 510, 92-101. [CrossRef]

55. Miura, T.; Muraoka, S.; Ogiso, T. Inhibition of lipid peroxidation by estradiol and 2-hydroxyestradiol. Steroids 1996, 61, 379-383. [CrossRef]

56. Patel, R.S.; Shah, G. High-fat diet exposure from pre-pubertal age induces polycystic ovary syndrome (PCOS) in rats. Reproduction 2018, 155, 139-149. [CrossRef]

57. Shim, J.; Shin, Y.; Lee, I.; Kim, S.Y. L-Methionine Production. Adv. Biochem. Eng. Biotechnol. 2017, 159, $153-177$.

58. Bauerle, M.R.; Schwalm, E.L.; Booker, S.J. Mechanistic Diversity of Radical S-Adenosylmethionine (SAM)-dependent Methylation. J. Biol. Chem. 2015, 290, 3995-4002. [CrossRef] [PubMed]

59. Lever, M.; Slow, S. The clinical significance of betaine, an osmolyte with a key role in methyl group metabolism. Clin. Biochem. 2010, 43, 732-744. [CrossRef]

60. Blachier, F.; Andriamihaja, M.; Blais, A. Sulfur-Containing Amino Acids and Lipid Metabolism. J. Nutr. 2020, 150 (Suppl. S1), 2524S-2531S. [CrossRef]

61. Shen, W.; Gao, C.; Cueto, R.; Liu, L.; Fu, H.; Shao, Y.; Yang, W.Y.; Fang, P.; Choi, E.T.; Wu, Q.; et al. Homocysteine-methionine cycle is a metabolic sensor system controlling methylation-regulated pathological signaling. Redox Biol. 2020, 28, 101322. [CrossRef] [PubMed]

62. Sanz-Fernandez, M.V.; Torres-Rovira, L.; Pesantez-Pacheco, J.L.; Vazquez-Gomez, M.; Garcia-Contreras, C.; Astiz, S.; GonzalezBulnes, A. A Cross-Sectional Study of Obesity Effects on the Metabolomic Profile of a Leptin-Resistant Swine Model. Metabolites 2020, 10, 89. [CrossRef] 